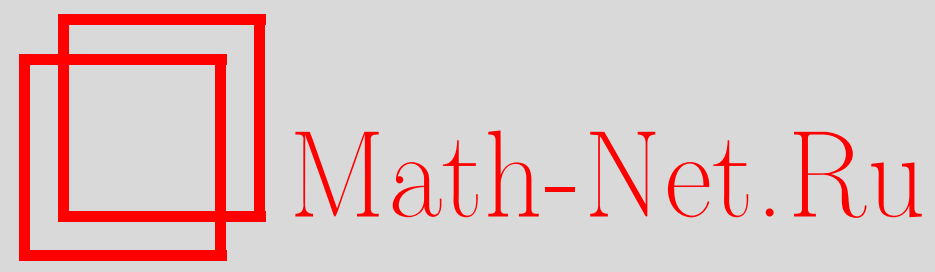

Д. В. Пильщиков, Анализ сложности алгоритма параллельного поиска "золотой" коллизии, Матем. вопр. криптогр., 2015, том 6, выпуск 4, 77-97

DOI: https://doi.org/10.4213/mvk169

Использование Общероссийского математического портала Math-Net.Ru подразумевает, что вы прочитали и согласны с пользовательским соглашением

http://www . mathnet.ru/rus/agreement

Параметры загрузки:

IP : 54.224 .187 .69

26 апреля 2023 г., 12:12:19

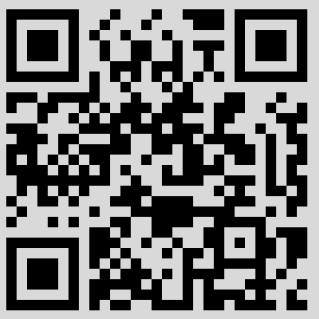


УДК: 519.719.2+519.218.2

\title{
Анализ сложности алгоритма параллельного поиска «золотой» коллизии
}

\author{
Д. В. Пильщиков \\ Лаборатория ТВП, Москва
}

Получено 20.IV.2015

В статье уточняются полученные ранее Оршотом и Винером оценки временно́й и емкостно́й сложностей предложенного ими алгоритма поиска «золотой» коллизии. Для этого используются результаты, относящиеся к расчету характеристик метода балансировки время-память-данные с особыми точками. Описывается вероятностная модель, позволяющая аппроксимировать исследуемые характеристики алгоритма случайными величинами, зависящими от числа частиц и суммарного числа частиц в докритическом процессе Гальтона-Ватсона. Найдены предельные значения математических ожиданий этих случайных величин.

Ключевые слова: поиск «золотой» коллизии, балансировка времяпамять-данные с особыми точками, ветвящиеся процессы, обращение однонаправленной функции

A complexity analysis of algorithm of parallel search of the "gold" collision D. V. Pilshchikov

TVP Laboratory, Moscow

\begin{abstract}
The paper refines known estimates of time and memory complexities of Oorschot and Wiener algorithm for the "gold" collision searching. We use results related to the computation of characteristics of time-memory-data tradeoff method with distinguished points. Probabilistic approximations of the algorithm characteristics by random variables depending on the number of particles and the total number of particles in a subcritical Galton-Watson process are described.The limits of expectations of these random variables are found.
\end{abstract}

Key words: "gold" collision search, time-memory-data tradeoff with distinguished points, branching processes, one-way function inversion

Citation: Mathematical Aspects of Cryptography, 2015, vol. 6, no. 4, pp. 77-97 (Russian). 
Методы поиска коллизии составляют важный раздел современного криптоанализа. Многие криптоаналитические проблемы (например, вычисление дискретного логарифма в циклических группах, нахождение коллизий криптографических хеш-функций, применение метода «встречи посередине») могут быть сведены к задаче поиска двух различных входов $x_{1}$ и $x_{2}$ функции $F$, удовлетворяющих соотношению $F\left(x_{1}\right)=F\left(x_{2}\right)$. В $[1,2]$ предложен параллельный переборный алгоритм решения этой задачи.

В этих работах выделено два случая: задача построения «небольшого» числа случайных коллизий функции $F$ и задача построения «большого» числа коллизий функции $F$ с последующим поиском среди них «золотой» коллизии (т.е. коллизии, позволяющей решить исходную криптографическую задачу). К первому случаю сводятся проблемы вычисления дискретного логарифма в циклических группах и нахождения коллизий криптографических хешфункций. Ко второму случаю сводится метод «встречи посередине», а также еще несколько задач (см. [3]). Здесь уточняются полученные в [1] оценки временно́й и емкостно́й сложностей предложенного в этой работе алгоритма поиска «золотой» коллизии.

\section{1. Постановка задачи}

Рассмотрим следующую задачу (о восстановлении ключа). Пусть

$$
B_{1}=E_{k_{2}}\left(E_{k_{1}}\left(A_{1}\right)\right), B_{2}=E_{k_{2}}\left(E_{k_{1}}\left(A_{2}\right)\right)
$$

- пара значений шифрованного текста, полученных из открытых текстов $A_{1}$ и $A_{2}$ с помощью композиции двух функций шифрования $E_{k_{1}}$ и $E_{k_{2}}$ посредством ключей $k_{1}$ и $k_{2}$. Для простоты предполагается, что открытые тексты, шифрованные тексты и ключи принадлежат множеству $X=\{1,2, \ldots, N\}$ :

$$
A_{1}, A_{2}, B_{1}, B_{2}, k_{1}, k_{2} \in X
$$

Требуется по двум парам открытого и шифрованного текстов $\left(A_{1}, B_{1}\right)$, $\left(A_{2}, B_{2}\right)$ восстановить пару ключей $\left(k_{1}, k_{2}\right)$.

Для решения данной задачи рассмотрим две функции

$$
\tilde{F}_{1}(x)=E_{x}\left(A_{1}\right), \tilde{F}_{2}(x)=E_{x}^{-1}\left(B_{1}\right),
$$

$l$ произвольных равновероятных функций

$$
\alpha_{i}: X \rightarrow\{1,2\}, i \in\{1, \ldots, l\}
$$


и $l$ произвольных биективных функций

$$
R_{i}: X \rightarrow X, i \in\{1, \ldots, l\}
$$

По формуле

$$
F_{i}(x)=R_{i}\left(\tilde{F}_{\alpha_{i}(x)}(x)\right)
$$

зададим $l$ функций $F_{i}: X \rightarrow X, i \in\{1, \ldots, l\}$.

Теперь для решения задачи восстановления ключа достаточно решить задачу поиска «золотой» коллизии, которая заключается в следующем. Требуется найти коллизию одной из функций $F_{i}, i \in \overline{1, l}$, например, $F_{i_{0}}\left(k_{1}\right)=$ $=F_{i_{0}}\left(k_{2}\right)$, удовлетворяющую «золотому» правилу

$$
\begin{gathered}
\alpha_{i_{0}}\left(k_{1}\right)=1, \alpha_{i_{0}}\left(k_{2}\right)=2, \\
E_{k_{1}}\left(A_{2}\right)=E_{k_{2}}^{-1}\left(B_{2}\right) .
\end{gathered}
$$

Для решения данной задачи к каждой из функций $F_{i}: X \rightarrow X, i \in \overline{1, l}$, применяется алгоритм параллельного поиска коллизий, и для каждой найденной коллизии проверяется выполнение «золотого» правила. Используемый при этом алгоритм параллельного поиска коллизий произвольной функции

$$
F: X \rightarrow X
$$

состоит из двух этапов: этапа построения таблицы (набора записей) и этапа построения коллизий.

Перед выполнением алгоритма выбираются два числа $t_{0}$ и $t_{1}, t_{0}<t_{1}$, множества начальных $S \subset X,|S|=m$, и особых $\Theta \subset X,|\Theta|<N$, точек.

На этапе построения таблицы для каждой начальной точки $x_{j, 0} \in S$, $j \in 1, \ldots, m$, путем последовательного применения функции $F$ вычисляется цепочка элементов

$$
x_{j, k+1}=F\left(x_{j, k}\right), k=0,1, \ldots
$$

Обрыв цепочки вычислений с началом в $x_{j, 0}$ происходит в точке $x_{j, \tau_{j}}$, $\tau_{j} \geq 1$, удовлетворяющей одному из двух условий:

$-x_{j, \tau_{j}}$ впервые попадает в множество особых точек $x_{j, \tau_{j}} \in \Theta$;

- длина цепочки $\tau_{j}$ достигает максимально разрешенной величины $\tau_{j}=t_{1}$.

Если цепочка оборвалась в связи с достижением особой точки и ее длина превышает минимально допустимое значение $t_{0}<\tau_{j}$, то тройка $\left(x_{j, 0}, x_{j, \tau_{j}}, \tau_{j}\right)$ записывается в таблицу. В этом случае цепочку будем называть табличной и говорить, что она соответствует записи $\left(x_{j, 0}, x_{j, \tau_{j}}, \tau_{j}\right)$. 
В основе вычислений, выполняемых на этапе построения коллизий функции $F$, лежит следующий факт.

Пусть у двух троек построенной таблицы, например $\left(x_{j_{1}, 0}, x_{j_{1}, \tau_{1}}, \tau_{j_{1}}\right)$ и $\left(x_{j_{2}, 0}, x_{j_{2}, \tau_{2}}, \tau_{j_{2}}\right), \tau_{j_{1}} \leq \tau_{j_{2}}$, совпадают вторые элементы: $x_{j_{1}, \tau_{j_{1}}}=x_{j_{2}, \tau_{j_{2}}}=$ $=\theta \in \Theta$. Это означает, что соответствующие им цепочки за некоторое количество тактов $k, 0 \leq k \leq \tau_{j_{1}}$, до своего конца $\theta$ сливаются и далее идут вместе. Следовательно, если $k<\tau_{j_{1}}$, то точки $x_{j_{1}, \tau_{j_{1}}-(k+1)}$ и $x_{j_{2}, \tau_{j_{2}}-(k+1)}$ образуют коллизию функции $F$.

Исходя из данного факта, на этапе построения коллизий функции $F$ выполняются следующие действия. Записи таблицы сортируются и разбиваются на группы с одинаковым значением второго элемента $\theta \in \Theta$. Далее, для каждой группы, содержащей более одной записи, частично восстанавливаются цепочки, соответствующие записям группы. Восстановление цепочек ведется последовательно. Если максимальная и минимальная длины цепочек рассматриваемой группы равны $\tau_{\max }$ и $\tau_{\min }$, то сначала строится множество точек $A(k)$, лежащих на цепочках за $k, k=\tau_{\max }$, тактов до их конца. Далее последовательно вычисляются множества $A\left(\tau_{\max }-1\right), A\left(\tau_{\max }-2\right), \ldots$ и т. д. Обрыв вычислений происходит при $k=k_{0}$, как только впервые выполняются условия $k_{0}<\tau_{\min }$ и $\left|A_{k_{0}}\right|=1$ (легко заметить, что при $k \leq k_{0}$ все цепочки группы сливаются в одну цепочку и дальнейший поиск коллизий не имеет смысла). В течение процесса построения множеств $A\left(\tau_{\max }-1\right), \ldots, A\left(k_{0}\right)$ очевидным образом определяется факт слияния цепочек и вычисляются коллизии функции $F$.

Точки из множеств $A\left(k_{0}-1\right), \ldots, A(1)$ будем называть специальными точками. Заметим, что они вычисляются на этапе построения таблицы, но не вычисляются на этапе построения коллизий.

Описанный выше алгоритм поиска «золотой» коллизии имеет следующие параметры:

$N$ - мощность множества $X$;

$t=\frac{N}{|\Theta|}-$ величина, обратная к доле особых точек среди всех точек множества $X$;

$m$ - число цепочек, генерируемых для построения одной таблицы;

$t_{0}, t_{1}$ - ограничения длины цепочки;

$l$ - количество используемых таблиц.

Данные параметры определяют надежность, временну́ю и емкостну́ю сложности (далее - характеристики) алгоритма поиска «золотой» коллизии, которые в рамках некоторой вероятностной модели задаются следующими величинами: 
$\eta$ - вероятность успешного завершения алгоритма;

$T_{0}$ - среднее суммарное количество вычислений функции $F$ на этапе построения таблиц;

$T_{1}$ - среднее суммарное количество вычислений функции $F$ на этапе построения коллизий;

$T$ - суммарная трудоемкость алгоритма $\left(T=T_{0}+T_{1}\right)$;

$M$ - размер памяти (среднее количество записей в таблице).

В [1] приводятся грубые оценки зависимости некоторых из указанных характеристик от параметров алгоритма. В частности, указывается, что величина $T$ имеет порядок $O\left(\sqrt{N^{3} / M}\right)$, в то время как при традиционном использовании метода «встречи посередине» она имеет порядок $O\left(\frac{N^{2}}{M}\right)$.

Новые результаты, полученные после 2000 года в области расчета характеристик метода Хеллмана, позволяют провести более детальный анализ зависимости характеристик алгоритма поиска «золотой коллизии» от его параметров. Это связано с тем, что указанный выше процесс построения таблиц по существу совпадает с процессом построения таблиц в методе Хеллмана с особыми точками $[4,5]$.

Вероятностная модель, предложенная в [6], позволяет строить модели исследуемых характеристик как случайных величин, зависящих от числа частиц и от суммарного числа частиц в докритическом процессе ГальтонаВатсона. Теоремы о предельном поведении этих случайных величин, приведенные в [6] и [7], позволяют получить оценки средних значений исследуемых характеристик, которые хорошо соответствуют экспериментальным данным при достаточно больших значениях параметров.

\section{2. Представление характеристик алгоритма при использовании одной таблицы}

Пусть $F: X \rightarrow X-$ фиксированное отображение и $F^{(k)}-$ его $k$-кратная композиция

$$
F^{(k)}(x)=\underbrace{F \circ F \circ \ldots \circ F}_{k @ 07}(x), F^{(0)}(x)=x .
$$

Для любой точки $x \in X$ через $D(x ; k)$ обозначим множество точек полного прообраза $x$ относительно $F^{(k)}$, удовлетворяющих дополнительным условиям:

$$
D(x ; k)=\left\{x^{\prime} \in X \mid F^{(k)}\left(x^{\prime}\right)=x, F^{(i)}\left(x^{\prime}\right) \notin\{\Theta \cup x\}, i \in\{0, \ldots, k-1\}\right\} .
$$


Пусть

$$
D\left(x ; k_{0}, k_{1}\right)=\bigcup_{k=k_{0}}^{k_{1}-1} D(x ; k)
$$

Предполагая, что каждый элемент множества $X$ независимо от других с вероятностью $m / N$ является началом цепочки, подсчитаем среднее значение некоторых случайных величин, являющихся моделями характеристик алгоритма поиска коллизий.

В разделе 2 случайные величины будем отмечать подчеркиванием снизу, а обозначения их средних значений - чертой сверху, например $\bar{\xi}=\mathbf{E} \underline{\xi}$.

Случайное множество начал цепочек обозначим символом $\underline{S} \subset X$. Отметим, что в используемой вероятностной модели для любого фиксированного множества $A \subset X$ выполняются соотношения

$$
\mathbf{E}|A \cap \underline{S}|=\frac{m}{N}|A|, \quad \mathbf{E} I\{A \cap \underline{S}=\emptyset\}=\left(1-\frac{m}{N}\right)^{|A|} .
$$

\section{1. Количество записей в таблице}

Количество записей в таблице обозначим символом $\underline{M}$. Оно равно количеству табличных цепочек. Количество табличных цепочек, заканчивающихся в особой точке $\theta \in \Theta$, равно количеству начал цепочек, лежащих в множестве $D\left(\theta ; t_{0}, t_{1}\right)$. Перечисляя все особые точки, получаем формулы

$$
\begin{gathered}
\underline{M}=\sum_{\theta \in \Theta}\left|D\left(\theta ; t_{0}, t_{1}\right) \cap \underline{S}\right|, \\
\bar{M}=\mathbf{E} \underline{M}=\frac{m}{N} \sum_{\theta \in \Theta}\left|D\left(\theta ; t_{0}, t_{1}\right)\right| .
\end{gathered}
$$

\section{2. Количество найденных коллизий}

Пусть $q-$ количество коллизий отображения $F$, которые найдены на этапе построения коллизий. Предположим, что в особой точке $\theta \in \Theta$ закончились $i>1$ табличных цепочек. Сливаясь, две цепочки дают одну коллизию. Следовательно, по записям таблицы, содержащим точку $\theta$, можно найти $i-1$ коллизий (для простоты считаем, что если в одной точке сливается $j>2$ цепочек, то в алгоритме используются только $j-1$ из $j(j-1) / 2$ возникающих в этой ситуации коллизий). Учитывая все особые точки, получаем, что 
суммарное количество построенных коллизий равно разнице между количеством всех табличных цепочек и количеством особых точек, через которые проходит хотя бы одна цепочка,

$$
\underline{q}=\underline{M}-\sum_{\theta \in \Theta}\left(1-I\left(\theta ; t_{0}, t_{1}\right)\right)
$$

где

$$
I\left(\theta ; t_{0}, t_{1}\right)=I\left\{D\left(\theta ; t_{0}, t_{1}\right) \cap \underline{S}=\varnothing\right\} .
$$

Отсюда получаем

$$
\bar{q}=\mathbf{E} q=\bar{M}-\sum_{\theta \in \Theta}\left(1-J\left(\theta ; t_{0}, t_{1}\right)\right)
$$

где

$$
J\left(y ; k_{0}, k_{1}\right)=\left(1-\frac{m}{N}\right)^{\left|D\left(y ; k_{0}, k_{1}\right)\right|} .
$$

\section{3. Суммарная длина цепочек, вычисленных на этапе построения таблиц}

Обозначим суммарную длину цепочек, вычисленных на этапе построения таблиц, символом $\underline{T}_{0}$. Если цепочка заканчивается в особой точке $\theta \in \Theta$ и начинается в множестве $D(\theta ; l), l<t_{1}$, то ее длина равна $l$. Для всех остальных цепочек длина равна $t_{1}$. Отсюда получаем формулы

$$
\begin{gathered}
\underline{T}_{0}=\sum_{\theta \in \Theta} \sum_{l=1}^{t_{1}} l|D(\theta ; l) \cap \underline{S}|+t_{1} \cdot\left|\left\{X \backslash \coprod_{\theta \in \Theta} D\left(\theta ; 0, t_{1}\right)\right\} \cap \underline{S}\right|, \\
\bar{T}_{0}=\mathbf{E} \underline{T}_{0}=\sum_{\theta \in \Theta} \sum_{l=1}^{t_{1}} l \frac{m}{N}|D(\theta ; l)|+t_{1} \frac{m}{N}\left(N-\sum_{\theta \in \Theta}\left|D\left(\theta ; 0, t_{1}\right)\right|\right) .
\end{gathered}
$$

\section{4. Количество точек, лежащих на табличных цепочках}

Количество точек, лежащих на табличных цепочках, обозначим символом $\underline{T}_{2}$. Пусть $\theta \in \Theta$ и $x^{\prime} \in D(\theta ; k), 0<k \leq t_{0}$. Тогда точка $x^{\prime}$ лежит на табличной цепочке, если хотя бы одно начало цепочек лежит в множестве $D\left(x^{\prime} ; t_{0}-k ; t_{1}-k\right)$. Аналогично, точка $x^{\prime} \in D(\theta ; k), t_{0}<k<t_{1}$, лежит на 
табличной цепочке, если хотя бы одно начало цепочек лежит в множестве $D\left(x^{\prime} ; 0 ; t_{1}-k\right)$. Отсюда получаем формулы

$$
\begin{gathered}
\underline{T}_{2}=\sum_{\theta \in \Theta} \sum_{k=1}^{t_{1}} \sum_{x^{\prime} \in D(\theta ; k)}\left(1-I\left(x^{\prime} ; 0 \vee\left(t_{0}-k\right), t_{1}-k\right)\right), \\
\bar{T}_{2}=\mathbf{E} \underline{T}_{2}=\sum_{\theta \in \Theta} \sum_{k=1}^{t_{1}} \sum_{x^{\prime} \in D(\theta ; k)}\left(1-J\left(x^{\prime} ; 0 \vee\left(t_{0}-k\right), t_{1}-k\right)\right),
\end{gathered}
$$

где через $a \vee b$ обозначен максимум $a$ и $b$.

\section{5. Количество специальных точек}

Количество специальных точек обозначим символом $\underline{T}_{3}$. Пусть $\theta \in \Theta$. Точка $x^{\prime} \in D(\theta ; k), 0<k \leq t_{0}$, является специальной, если она лежит на табличной цепочке и ни одна из точек множества $D(\theta ; k) \backslash x^{\prime}$ не лежит на табличной цепочке. Точка $x^{\prime} \in D(\theta ; k), t_{0}<k \leq t_{1}$, является специальной, если она лежит на табличной цепочке, ни одна из точек множества $D(\theta ; k) \backslash x^{\prime}$ не лежит на табличной цепочке и длина любой табличной цепочки, заканчивающейся в $\theta$, больше $k$.

Отсюда получаем формулы

$$
\begin{aligned}
& \underline{T}_{3}= \sum_{\theta \in \Theta} \sum_{k=1}^{t_{0}} \sum_{x^{\prime} \in D(\theta ; k)}\left(1-I\left(x^{\prime} ; t_{0}-k, t_{1}-k\right)\right) \times \\
& \times \prod_{x^{\prime \prime} \in\left\{D(\theta ; k) \backslash x^{\prime}\right\}} I\left(x^{\prime \prime} ; t_{0}-k, t_{1}-k\right)+ \\
&+\sum_{\theta \in \Theta} \sum_{k=t_{0}+1}^{t_{1}} \sum_{x^{\prime} \in D(\theta ; k)} I\left(\theta ; t_{0}, k\right)\left(1-I\left(x^{\prime} ; 0, t_{1}-k\right)\right) \times \\
& \quad \times \prod_{x^{\prime \prime} \in\left\{D(\theta ; k) \backslash x^{\prime}\right\}} I\left(x^{\prime \prime} ; 0, t_{1}-k\right) . \\
& \bar{T}_{3}=\mathbf{E} \underline{T}_{3}=\sum_{\theta \in \Theta} \sum_{k=1}^{t_{0}} \sum_{x^{\prime} \in D(\theta ; k)}\left(1-J\left(x^{\prime} ; t_{0}-k, t_{1}-k\right)\right) \times \\
& \quad \times \prod_{x^{\prime \prime} \in\left\{D(\theta ; k) \backslash x^{\prime}\right\}} J\left(x^{\prime \prime} ; t_{0}-k, t_{1}-k\right)+
\end{aligned}
$$




$$
\begin{gathered}
+\sum_{\theta \in \Theta} \sum_{k=t_{0}+1}^{t_{1}} \sum_{\substack{x^{\prime} \in D(\theta ; k) \\
\times}} J\left(\theta ; t_{0}, k\right)\left(1-J\left(x^{\prime} ; 0, t_{1}-k\right)\right) \times \\
\times \prod_{x^{\prime \prime} \in\left\{D(\theta ; k) \backslash x^{\prime}\right\}} J\left(x^{\prime \prime} ; 0, t_{1}-k\right) .
\end{gathered}
$$

\section{6. Количество точек, вычисляемых на этапе построения коллизий}

Количество точек, вычисляемых на этапе построения коллизий, обозначим символом $\underline{T}_{1}$. На этапе построения коллизий происходит однократное вычисление всех точек, лежащих на табличных цепочках, за исключением специальных, следовательно,

$$
\underline{T}_{1}=\underline{T}_{2}-\underline{T}_{3}, \quad \bar{T}_{1}=\bar{T}_{2}-\bar{T}_{3} .
$$

\section{3. Представление характеристик с помощью средних значений случайных величин, зависящих от числа частиц и суммарного числа частиц в процессе Гальтона - Ватсона}

Пусть теперь $F-$ случайное отображение. Так как значения $\bar{M}, \bar{q}, \bar{T}_{0}$, $\bar{T}_{1}$, определенные формулами (1)-(6), зависят от $F$, то они становятся случайными величинами. Опишем подход, в рамках которого получим оценки $\hat{M}, \hat{q}$, $\hat{T}_{0}, \hat{T}_{1}$ средних значений данных случайных величин.

Для простоты будем пренебрегать зависимостью между случайными величинами $|D(x ; 1)|, x \in X$, равными количествам непосредственных прообразов точек $x$ из множества $X \backslash\{\Theta \cup x\}$ относительно отображения $F$, и считать, что они независимы в совокупности.

В этом случае распределение случайных величин вида $|D(x ; k)|, x \in X$, $0 \leq k \leq t_{1}$, совпадает с распределением числа $\mu(k)$ частиц, существующих в момент времени $k$ в процессе Гальтона-Ватсона $\mu(i), i=0,1, \ldots$, определяемом соотношениями

$$
\mu(0)=1, \quad \mathbf{P}(\mu(1)=j)=\mathbf{P}(|D(x ; 1)|=j), \quad j=0,1, \ldots
$$

(распределение в правой части индуцируется $F$ и не зависит от $x$ ). 
Можно показать, что первый момент случайной величины $\mu(1)$ равен $1-\frac{1}{t}\left(t=\frac{N}{|\Theta|}\right)$, а два ее следующих факториальных момента при $N, t \rightarrow \infty$ стремятся к 1 .

Аналогично, используя соотношение $\left|D\left(x ; k_{0}, k_{1}\right)\right|=\sum_{k=k_{0}}^{k_{1}-1}|D(x ; k)|$, замечаем, что при сделанном предположении распределение случайных величин вида $\left|D\left(x ; k_{0}, k_{1}\right)\right|, x \in X, 0 \leq k_{0} \leq k_{1} \leq t_{1}$, совпадает с распределением суммарного числа частиц

$$
v\left(k_{0}, k_{1}\right)=\sum_{i=k_{0}}^{k_{1}-1} \mu(i),
$$

существовавших в процессе Гальтона-Ватсона $\mu(i), i=0,1, \ldots$, в моменты времени $k_{0}, \ldots, k_{1}-1$.

В качестве оценок средних значений случайных величин $\bar{M}, \bar{q}, \bar{T}_{0}, \bar{T}_{1}$ рассмотрим средние значения случайных величин, полученных из формул (1)-(6) посредством замены случайных величин вида $|D(x ; k)|, 0 \leq k \leq$ $t_{1},\left|D\left(x ; k_{0}, k_{1}\right)\right|, 0 \leq k_{0} \leq k_{1} \leq t_{1}$, при любых $x \in X$ случайными величинами $\mu(k), v\left(k_{0}, k_{1}\right)$ соответственно.

Проведя необходимые вычисления, получаем следующие оценки:

$$
\begin{gathered}
\hat{M}=\frac{m}{N} \sum_{\theta \in \Theta} \mathbf{E} v\left(t_{0}, t_{1}\right)=\frac{m}{t} \mathbf{E} v\left(t_{0}, t_{1}\right), \\
\hat{q}=\hat{M}-\frac{N}{t}\left(1-\tilde{J}\left(t_{0}, t_{1}\right)\right), \\
\hat{T}_{0}=\frac{m}{t} \sum_{k=1}^{t_{1}} k \mathbf{E} \mu(k)+t_{1} m\left(1-\frac{\mathbf{E} v\left(0, t_{1}\right)}{t}\right), \\
\hat{T}_{3}=\frac{N}{t} \sum_{k=1}^{t_{0}}\left(1-\tilde{J}\left(t_{0}-k, t_{1}-k\right)\right) F_{k}\left(\tilde{J}\left(t_{0}-k, t_{1}-k\right)\right)+ \\
\frac{N}{t} \sum_{k=t_{0}+1}^{t_{1}}\left(1-\tilde{J}\left(0 \vee\left(t_{0}-k\right), t_{1}-k\right)\right) \mathbf{E} \mu(k), \\
\hat{T}_{1}=\hat{T}_{2}-\hat{T}_{3},
\end{gathered}
$$

где

$$
F_{k}(y)=\mathbf{E} \mu(k) y^{\mu(k)-1},
$$




$$
F_{k_{0}, k_{1}}(y, z)=\mathbf{E} \mu\left(k_{1}\right) y^{\mu\left(k_{1}\right)-1} z^{v\left(k_{0}, k_{1}\right)}
$$

\section{4. Вычисление предельных значений характеристик алгоритма при использовании одной таблицы}

Точное значение оценок $\hat{M}, \hat{q}, \hat{T}_{0}, \hat{T}_{1}$ сложным образом зависит от распределения процесса $\mu$. Тем не менее, как будет показано далее, при определенном асимптотическом изменении параметров $m, t, N, t_{0}, t_{1}$ величины $\hat{M}, \hat{q}, \hat{T}_{0}, \hat{T}_{1}$ сближаются со значениями некоторых функций, зависящих только от этих параметров. Будем использовать значения данных функций параметров в качестве итоговых характеристик алгоритма поиска коллизий, обозначать символами $M, q, T_{0}, T_{1}$ и называть их следующим образом:

$M$ - размер таблицы;

$q$ - количество коллизий, вычисленных по одной таблице;

$T_{0}$ - трудоемкость построения таблицы (количество вычислений значений функции $F$ на этапе построения таблицы);

$T_{1}$ - трудоемкость построения коллизий (количество вычислений значений функции $F$ на этапе построения коллизий).

Приведенная далее теорема 1 позволяет представить функции $M, q, T_{0}, T_{1}$ формулами

$$
\begin{gathered}
M=m \cdot \gamma_{0}\left(x_{0}, x_{1}\right), \quad x_{0}=t_{0} / t, \quad x_{1}=t_{1} / t \\
q=m \cdot \gamma_{1}\left(\alpha, x_{0}, x_{1}\right), \quad \alpha=\frac{m t^{2}}{2 N}, \\
T_{0}=m t \cdot \gamma_{2}\left(x_{1}\right), \\
T_{1}=m t \cdot \gamma_{3}\left(\alpha, x_{0}, x_{1}\right)
\end{gathered}
$$

и получить точный вид множителей $\gamma_{0}, \gamma_{1}, \gamma_{2}, \gamma_{3}$.

Для формулировки теоремы понадобятся определения нескольких функций:

$$
\begin{gathered}
f_{0}(y, z)=\frac{y+\sqrt{z} \cdot \tanh (\sqrt{z})}{1+y \cdot \frac{\tanh (\sqrt{z})}{\sqrt{z}}} \text { при } z>0, \quad f_{0}(y, 0)=\frac{y}{1+y}, \\
f_{1}(x, y, z)=\frac{1}{x} f_{0}\left(y x, z x^{2}\right) \text { при } x>0, \quad f_{1}(0, y, z)=y, \\
f(x, y, z)=2 f_{1}(x, y / 2+1 / 2, z / 2+1 / 4)-1,
\end{gathered}
$$




$$
\begin{gathered}
\tilde{f}(x, y, z)=\frac{\partial f(x, y, z)}{\partial y}, \\
\tilde{\tilde{f}}\left(x_{0}, x_{1}, y, z\right)=\frac{\partial f\left(x_{0}, f\left(x_{1}-x_{0}, y, z\right), 0\right)}{\partial y}= \\
\tilde{f}\left(x_{0}, f\left(x_{1}-x_{0}, y, z\right), 0\right) \tilde{f}\left(x_{1}-x_{0}, y, z\right) .
\end{gathered}
$$

Далее будем полагать, что для каждого натурального $s$ заданы набор параметров $N_{s}, m_{s}, t_{s}, t_{0, s}, t_{1, s}$, процесс Гальтона-Ватсона $\mu_{s}, \mu_{s}(0)=1, \mathbf{E} \mu_{s}(1)=1-$ $-\frac{1}{t_{s}}$, и по формулам (7)-(12) определены величины $\hat{M}_{s}, \hat{q}_{s}, \hat{T}_{0, s}, \hat{T}_{1, s}, \hat{T}_{2, s}, \hat{T}_{3, s}$.

Теорема 1. Пусть при $s \rightarrow \infty$ выполняются следуюшие асимптотические соотношения:

$$
\begin{gathered}
N_{s}, m_{s}, t_{s}, t_{0, s}, t_{1, s} \rightarrow \infty, \\
\frac{m_{s} t_{s}^{2}}{2 N_{s}} \rightarrow \alpha, \quad \frac{t_{0, s}}{t_{s}} \rightarrow x_{0}, \quad \frac{t_{1, s}}{t_{s}} \rightarrow x_{1}, \quad x_{0}<x_{1}, \\
\mathbf{E} \mu_{s}^{[2]}(1) \rightarrow 1, \quad \mathbf{E} \mu_{s}^{[3]}(1)<R<\infty .
\end{gathered}
$$

Тогда при $s \rightarrow \infty$ существуют предель

1) $\frac{\hat{M}_{s}}{m_{s}} \rightarrow \gamma_{0}\left(x_{0}, x_{1}\right), \gamma_{0}\left(x_{0}, x_{1}\right)=e^{-x_{0}}-e^{-x_{1}}$,

2) $\frac{\hat{q}_{s}}{m_{s}} \rightarrow \gamma_{1}\left(\alpha, x_{0}, x_{1}\right)$,

$$
\gamma_{1}\left(\alpha, x_{0}, x_{1}\right)=e^{-x_{0}}-e^{-x_{1}}-\frac{1}{2 \alpha} f\left(x_{0}, f\left(x_{1}-x_{0}, 0,2 \alpha\right), 0\right),
$$

3) $\frac{\hat{T}_{0, s}}{m_{s} t_{s}} \rightarrow \gamma_{2}\left(x_{1}\right), \gamma_{2}\left(x_{1}\right)=1-e^{-x_{1}}$,

4) $\frac{\hat{T}_{1, s}}{m_{s} t_{s}} \rightarrow \gamma_{3}\left(\alpha, x_{0}, x_{1}\right)$,

$$
\begin{aligned}
\gamma_{3}\left(\alpha, x_{0}, x_{1}\right)= & \frac{1}{2 \alpha} \int_{0}^{x_{0}} f\left(x_{0}-x, f\left(x_{1}-x_{0}, 0,2 \alpha\right), 0\right) \gamma_{3,1}\left(\alpha, x_{0}, x_{1}\right) d x+ \\
& +\frac{1}{2 \alpha} \int_{x_{0}}^{x_{1}} f\left(x_{1}-x, 0,2 \alpha\right) \gamma_{3,2}\left(\alpha, x_{0}, x_{1}\right) d x
\end{aligned}
$$

$\gamma_{3,1}\left(\alpha, x_{0}, x_{1}, x\right)=\left(e^{-x}-\tilde{f}\left(x, f\left(x_{0}-x, f\left(x_{1}-x_{0}, 0,2 \alpha\right), 0\right), 0\right)\right)$,

$$
\gamma_{3,2}\left(\alpha, x_{0}, x_{1}, x\right)=e^{-x}-\tilde{\tilde{f}}\left(x_{0}, x, f\left(x_{1}-x, 0,2 \alpha\right), 2 \alpha\right) .
$$




\section{Доказательство.}

1) Имеем представление

$$
\frac{\hat{M}_{s}}{m_{s}}=\frac{1}{t_{s}} \mathbf{E} v_{s}\left(t_{0, s}, t_{1, s}\right) .
$$

Согласно [7, теорема 1, п. 3] в условиях теоремы существует предел

$$
\frac{1}{t_{s}} \mathbf{E} v_{s}\left(i_{s}, j_{s}\right) \rightarrow e^{-x_{0}}-e^{-x_{1}}, \quad s \rightarrow \infty .
$$

Следовательно,

$$
\gamma_{0}\left(x_{0}, x_{1}\right)=e^{-x_{0}}-e^{-x_{1}} .
$$

2) Имеем представление

$$
\frac{\hat{q}_{s}}{m_{s}}=\frac{\hat{M}}{m_{s}}-\frac{N_{s}}{m_{s} t_{s}^{2}} \cdot t_{s}\left(1-\tilde{J}_{s}\left(t_{0, s}, t_{1, s}\right)\right) .
$$

Согласно [7, теорема 2, п. 4] в условиях теоремы существует предел

$$
\begin{gathered}
t_{s}\left(1-\tilde{J}_{s}\left(t_{0, s}, t_{1, s}\right)\right)= \\
t_{s}\left(1-\mathbf{E}\left(1-\frac{m_{s} t_{s}^{2}}{N_{s}} \cdot \frac{1}{t_{s}^{2}}\right)^{v_{s}\left(t_{0, s}, t_{1, s}\right)}\right) \rightarrow f\left(x_{0}, f\left(x_{1}-x_{0}, 0,2 \alpha\right), 0\right), \quad s \rightarrow \infty .
\end{gathered}
$$

Следовательно,

$$
\gamma_{1}\left(\alpha, x_{0}, x_{1}\right)=\gamma_{0}\left(x_{0}, x_{1}\right)-\frac{1}{2 \alpha} f\left(x_{0}, f\left(x_{1}-x_{0}, 0,2 \alpha\right), 0\right) .
$$

3) Имеем представление

$$
\frac{\hat{T}_{0}}{m_{s} t_{s}}=\sum_{k=1}^{t_{1, s}} \frac{k}{t_{s}} \mathbf{E} \mu_{s}(k) \frac{1}{t_{s}}+\frac{t_{1, s}}{t_{s}}\left(1-\frac{\mathbf{E} v_{s}\left(0, t_{1, s}\right)}{t_{s}}\right) .
$$

Используя известную формулу $\mathbf{E} \mu_{s}(k)=\left(1-\frac{1}{t_{s}}\right)^{k}$, первое слагаемое суммы (14) представляем в виде интеграла от ступенчатой функции

$$
\sum_{k=1}^{t_{1, s}} \frac{k}{t_{s}} \mathbf{E} \mu_{s}(k) \frac{1}{t_{s}}=\int_{0}^{t_{1, s} / t_{s}} \frac{\left[x t_{s}\right]}{t_{s}}\left(1-\frac{1}{t_{s}}\right)^{t_{s} \cdot \frac{\left[x t_{s}\right]}{t_{s}}} d x .
$$


Подынтегральные функции ограничены в совокупности и поточечно сходятся к пределу

$$
\frac{\left[x t_{s}\right]}{t_{s}}\left(1-\frac{1}{t_{s}}\right)^{t_{s} \cdot \frac{\left[x t_{s}\right]}{t_{s}}} \rightarrow x e^{-x}, \quad s \rightarrow \infty,
$$

следовательно, имеет место сходимость

$$
\sum_{k=1}^{t_{1, s}} \frac{k}{t_{s}} \mathbf{E} \mu_{s}(k) \frac{1}{t_{s}} \rightarrow \int_{0}^{x_{1}} x e^{-x} d x=1-\left(x_{1}+1\right) e^{-x_{1}}, \quad s \rightarrow \infty .
$$

Предел второго слагаемого суммы (14) находим с помощью соотношения

$$
\frac{t_{1, s}}{t_{s}}\left(1-\frac{\mathbf{E} v_{s}\left(0, t_{1, s}\right)}{t_{s}}\right) \rightarrow x_{1}\left(1-\gamma_{0}\left(0, x_{1}\right)\right)=x_{1} e^{-x_{1}}, \quad s \rightarrow \infty .
$$

Собирая формулы (15) и (16) воедино, получаем $\gamma_{2}\left(x_{1}\right)=1-e^{-x_{1}}$.

4) Вычислим предел последовательности $\frac{\hat{T}_{2, s}}{m_{s} t_{s}}, s=1,2, \ldots$ Имеем представление

$$
\begin{gathered}
\frac{\hat{T}_{2, s}}{m_{s} t_{s}}=\frac{N_{s}}{m_{s} t_{s}^{2}} \sum_{k=1}^{t_{0, s}} t_{s}\left(1-\tilde{J}_{s}\left(t_{0, s}-k, t_{1, s}-k\right)\right) \mathbf{E} \mu_{s}(k) \frac{1}{t_{s}}+ \\
\quad+\frac{N_{s}}{m_{s} t_{s}^{2}} \sum_{k=t_{0}+1}^{t_{1, s}} t_{s}\left(1-\tilde{J}_{s}\left(0, t_{1, s}-k\right)\right) \mathbf{E} \mu_{s}(k) \frac{1}{t_{s}}= \\
=\frac{N_{s}}{m_{s} t_{s}^{2}} \int_{0}^{t_{0} / t_{s}} t_{s}\left(1-\tilde{J}_{s}\left(t_{0, s}-\left[x t_{s}\right], t_{1, s}-\left[x t_{s}\right]\right)\right) \mathbf{E} \mu_{s}\left(\left[x t_{s}\right]\right) d x+ \\
+\frac{N_{s}}{m_{s} t_{s}^{2}} \int_{t_{0, s} / t_{s}}^{t_{1, s} / t_{s}} t_{s}\left(1-\tilde{J}_{s}\left(0, t_{1, s}-\left[x t_{s}\right]\right)\right) \mathbf{E} \mu_{s}\left(\left[x t_{s}\right]\right) d x .
\end{gathered}
$$

Из оценок

$$
t_{s}\left(1-\left(1-\frac{m_{s}}{N_{s}}\right)^{v_{s}\left(t^{\prime}, t^{\prime \prime}\right)}\right) \leq \frac{v_{s}\left(t^{\prime}, t^{\prime \prime}\right)}{t_{s}} \frac{m_{s} t_{s}^{2}}{N_{s}}, \quad \frac{\mathbf{E} v_{s}\left(t^{\prime}, t^{\prime \prime}\right)}{t_{s}} \leq \frac{t^{\prime \prime}-t^{\prime}}{t_{s}},
$$

получаем ограниченность подынтегральных функций в совокупности. Из (13) при $s \rightarrow \infty$ следует поточечная сходимость

$$
t_{s}\left(1-\tilde{J}_{s}\left(t_{0, s}-\left[x t_{s}\right], t_{1, s}-\left[x t_{s}\right]\right)\right) \rightarrow f\left(x_{0}-x, f\left(x_{1}-x_{0}, 0,2 \alpha\right), 0\right),
$$




$$
\begin{gathered}
t_{s}\left(1-\tilde{J}_{s}\left(0, t_{1, s}-\left[x t_{s}\right]\right)\right) \rightarrow f\left(0, f\left(x_{1}-x, 0,2 \alpha\right), 0\right)=f\left(x_{1}-x, 0,2 \alpha\right), \\
\quad \mathbf{E} \mu_{s}\left(\left[x t_{s}\right]\right) \rightarrow e^{-x} .
\end{gathered}
$$

Отсюда получаем, что

$$
\begin{aligned}
\frac{\hat{T}_{2, s}}{m_{s} t_{s}} \rightarrow & \frac{1}{2 \alpha} \int_{0}^{x_{0}} f\left(x_{0}-x, f\left(x_{1}-x_{0}, 0,2 \alpha\right), 0\right) e^{-x} d x+ \\
& +\frac{1}{2 \alpha} \int_{x_{0}}^{x_{1}} f\left(x_{1}-x, 0,2 \alpha\right) e^{-x} d x, s \rightarrow \infty .
\end{aligned}
$$

Вычислим предел последовательности $\frac{\hat{T}_{3, s}}{m_{s} t_{s}}, s=1,2, \ldots$ Имеем представление

$$
\begin{gathered}
\frac{\hat{T}_{3, s}}{m_{s} t_{s}}=\frac{N_{s}}{m_{s} t_{s}^{2}} \sum_{k=1}^{t_{0, s}} t_{s}\left(1-\tilde{J}_{s}\left(t_{0, s}-k, t_{1, s}-k\right)\right) \cdot F_{k, s}\left(\tilde{J}_{s}\left(t_{0, s}-k, t_{1, s}-k\right)\right) \cdot \frac{1}{t_{s}}+ \\
+\frac{N_{s}}{m_{s} t_{s}^{2}} \sum_{k=t_{0, s}+1}^{t_{1, s}} t_{s}\left(1-\tilde{J}_{s}\left(0, t_{1, s}-k\right)\right) \cdot F_{t_{0, s}, k, s}\left(\tilde{J}_{s}\left(0, t_{1, s}-k\right), 1-\frac{m_{s}}{N_{s}}\right) \cdot \frac{1}{t_{s}}= \\
=\frac{N_{s}}{m_{s} t_{s}^{2}} \int_{0}^{t_{0, s} / t_{s}} t_{s}\left(1-\tilde{J}_{s}\left(t_{0, s}-\left[x t_{s}\right], t_{1, s}-\left[x t_{s}\right]\right)\right) \cdot \\
+\frac{N_{s}}{m_{s} t_{s}^{2}} \int_{t_{0, s} / t_{s}}^{t_{1, s} / t_{s}} t_{s}\left(1-\tilde{J}_{s}\left(0, t_{1, s}-\left[x t_{s}\right]\right)\right) \cdot F_{t_{0, s},\left[x t_{s}\right], s}\left(\tilde{J}_{s}\left(0, t_{1, s}-\left[x t_{s}\right]\right), 1-\frac{m_{s}}{N_{s}}\right) d x .
\end{gathered}
$$

Согласно [7, теорема 1, п. 11] из существования предела

$$
y_{s} \rightarrow y, \quad s \rightarrow \infty
$$

следует существование предела

$$
\mathbf{E} \mu_{s}\left(t_{0, s}\right)\left(1-\frac{y_{s}}{t_{s}}\right)^{\mu_{s}\left(t_{0, s}\right)-1}=F_{t_{0, s}, s}\left(1-\frac{y_{s}}{t_{s}}\right) \rightarrow \tilde{f}\left(x_{0}, y, 0\right), \quad s \rightarrow \infty,
$$


где $\tilde{f}(x, y, z)=\frac{\partial f(x, y, z)}{\partial y}$. Учитывая представление

$$
\tilde{J}_{s}\left(t_{0, s}-\left[x t_{s}\right], t_{1, s}-\left[x t_{s}\right]\right)=1-\frac{y_{s}}{t_{s}}, \quad y_{s}=t_{s}\left(1-\tilde{J}_{s}\left(t_{0, s}-\left[x t_{s}\right], t_{1, s}-\left[x t_{s}\right]\right)\right),
$$

при $s \rightarrow \infty$ получаем существование поточечного предела

$$
F_{\left[x t_{s}\right], s}\left(\tilde{J}_{s}\left(t_{0, s}-\left[x t_{s}\right], t_{1, s}-\left[x t_{s}\right]\right)\right) \rightarrow \tilde{f}\left(x, f\left(x_{0}-x, f\left(x_{1}-x_{0}, 0,2 \alpha\right), 0\right), 0\right) .
$$

В излагаемой ниже теореме 2 показано, что если существует предел

$$
y_{s} \rightarrow y, z_{s} \rightarrow z, s \rightarrow \infty
$$

то существует предел

$$
F_{t_{0, s}, t_{1, s, s}}\left(1-\frac{y_{s}}{t_{s}}, 1-\frac{z_{s}}{t_{s}^{2}}\right) \rightarrow \tilde{\tilde{f}}\left(x_{0}, x_{1}, y, z\right), \quad s \rightarrow \infty,
$$

где $\tilde{\tilde{f}}\left(x_{0}, x_{1}, y, z\right)=\frac{\partial f\left(x_{0}, f\left(x_{1}-x_{0}, y, z\right), 0\right)}{\partial y}$. Отсюда, учитывая сходимости

$$
\begin{gathered}
t_{s}\left(1-\tilde{J}_{s}\left(0, t_{1, s}-t_{0, s}\right)\right) \rightarrow f\left(x_{1}-x_{0}, 0,2 \alpha\right), \\
\frac{t_{s}^{2} m_{s}}{N_{s}} \rightarrow 2 \alpha,
\end{gathered}
$$

получаем поточечную сходимость функций

$$
F_{t_{0, s},\left[x t_{s}\right], s}\left(\tilde{J}_{s}\left(0, t_{1, s}-\left[x t_{s}\right]\right), 1-\frac{m_{s}}{N_{s}}\right) \rightarrow \tilde{\tilde{f}}\left(x_{0}, x, f\left(x_{1}-x, 0,2 \alpha\right), 2 \alpha\right), \quad s \rightarrow \infty .
$$

Подынтегральные функции в интегралах (17) ограничены в совокупности и сходятся поточечно, поэтому при $s \rightarrow \infty$ справедливы предельные переходы

$$
\begin{gathered}
\frac{N_{s}}{m_{s} t_{s}^{2}} \int_{0}^{t_{0, s} / t_{s}} t_{s}\left(1-\tilde{J}_{s}\left(t_{0, s}-\left[x t_{s}\right], t_{1, s}-\left[x t_{s}\right]\right)\right) \cdot \\
\cdot F_{\left[x t_{s}\right], s}\left(\tilde{J}_{s}\left(t_{0, s}-\left[x t_{s}\right], t_{1, s}-\left[x t_{s}\right]\right)\right) d x \rightarrow \\
\rightarrow \frac{1}{2 \alpha} \int_{0}^{x_{0}} f\left(x_{0}-x, f\left(x_{1}-x_{0}, 0,2 \alpha\right), 0\right) \cdot \tilde{f}\left(x, f\left(x_{0}-x, f\left(x_{1}-x_{0}, 0,2 \alpha\right), 0\right), 0\right) d x,
\end{gathered}
$$




$$
\begin{gathered}
\frac{N_{s}}{m_{s} t_{s}^{2}} \int_{t_{0, s} / t_{s}}^{t_{1, s} / t_{s}} t_{s}\left(1-\tilde{J}_{s}\left(0, t_{1, s}-\left[x t_{s}\right]\right)\right) \cdot F_{t_{0, s},\left[x t_{s}\right], s}\left(\tilde{J}_{s}\left(0, t_{1, s}-\left[x t_{s}\right]\right), 1-\frac{m_{s}}{N_{s}}\right) d x \rightarrow \\
\quad \rightarrow \frac{1}{2 \alpha} \int_{x_{0}}^{x_{1}} f\left(x_{1}-x, 0,2 \alpha\right) \cdot \tilde{\tilde{f}}\left(x_{0}, x, f\left(x_{1}-x, 0,2 \alpha\right), 2 \alpha\right) d x
\end{gathered}
$$

Учитывая соотношение $\frac{\hat{T}_{1, s}}{m_{s} t_{s}}=\frac{\hat{T}_{2, s}}{m_{s} t_{s}}-\frac{\hat{T}_{3, s}}{m_{s} t_{s}}$, находим

$$
\begin{aligned}
\gamma_{3}\left(\alpha, x_{0}, x_{1}\right)= & \frac{1}{2 \alpha} \int_{0}^{x_{0}} f\left(x_{0}-x, f\left(x_{1}-x_{0}, 0,2 \alpha\right), 0\right) \gamma_{3,1}\left(\alpha, x_{0}, x_{1}, x\right) d x+ \\
& +\frac{1}{2 \alpha} \int_{x_{0}}^{x_{1}} f\left(x_{1}-x, 0,2 \alpha\right) \gamma_{3,2}\left(\alpha, x_{0}, x_{1}, x\right) d x,
\end{aligned}
$$

где

$$
\begin{gathered}
\gamma_{3,1}\left(\alpha, x_{0}, x_{1}, x\right)=\left(e^{-x}-\tilde{f}\left(x, f\left(x_{0}-x, f\left(x_{1}-x_{0}, 0,2 \alpha\right), 0\right), 0\right)\right), \\
\gamma_{3,2}\left(\alpha, x_{0}, x_{1}, x\right)=\left(e^{-x}-\tilde{\tilde{f}}\left(x_{0}, x, f\left(x_{1}-x, 0,2 \alpha\right), 2 \alpha\right)\right) .
\end{gathered}
$$

Теорема доказана.

При доказательстве теоремы 1 использовалось следующее утверждение.

Теорема 2. Пусть при $s \rightarrow \infty$ выполняются асимптотические соотношения

$$
\begin{gathered}
t_{s}, t_{0, s}, t_{1, s} \rightarrow \infty, \\
\frac{t_{0, s}}{t_{s}} \rightarrow x_{0}, \quad \frac{t_{1, s}}{t_{s}} \rightarrow x_{1}, \quad x_{0}<x_{1}, \quad y_{s} \rightarrow y, \quad z_{s} \rightarrow z, \\
\mathbf{E} \mu_{s}^{[2]}(1) \rightarrow 1, \quad \mathbf{E} \mu_{s}^{[3]}(1)<R<\infty .
\end{gathered}
$$

Тогда существует предел

$$
\begin{aligned}
& \mathbf{E} \mu_{s}\left(t_{1, s}\right)\left(1-\frac{y_{s}}{t_{s}}\right)^{\mu_{s}\left(t_{1, s}\right)-1}\left(1-\frac{z_{s}}{t_{s}^{2}}\right)^{v_{s}\left(t_{0, s}, t_{1, s}\right)} \rightarrow \tilde{\tilde{f}}\left(x_{0}, x_{1}, y, z\right), \quad s \rightarrow \infty, \\
& \text { где } \tilde{\tilde{f}}\left(x_{0}, x_{1}, y, z\right)=\frac{\partial f\left(x_{0}, f\left(x_{1}-x_{0}, y, z\right), 0\right)}{\partial y} .
\end{aligned}
$$


Доказательство. Пользуясь известными свойствами процесса Гальтона-Ватсона $\mu$, легко показать, что для любых $y, z \geq 0$ верны представления

$$
\begin{gathered}
\mathbf{E} \mu\left(k_{1}\right)\left(1-\frac{y}{t}\right)^{\mu\left(k_{1}\right)-1}\left(1-\frac{z}{t^{2}}\right)^{v\left(k_{0}, k_{1}\right)}=\frac{\partial \bar{F}_{k_{0}, k_{1}}\left(1-\frac{y}{t}, 1-\frac{z}{t^{2}}\right)}{\partial y} \\
\bar{F}_{k_{0}, k_{1}}\left(1-\frac{y}{t}, 1-\frac{z}{t^{2}}\right)=t\left(1-E\left(1-\frac{y}{t}\right)^{\mu\left(k_{1}\right)}\left(1-\frac{z}{t^{2}}\right)^{v\left(k_{0}, k_{1}\right)}\right)= \\
=t\left(1-G_{k_{0}}\left(G_{k_{1}-k_{0}}\left(1-\frac{y}{t}, 1-\frac{z}{t^{2}}\right), 1\right)\right) \\
G_{k}(y, z)=\mathbf{E} y^{\mu(k)} z^{v(0, k)}
\end{gathered}
$$

Согласно [6] в рамках условий теоремы существует предел

$$
t_{s}\left(1-G_{\left[x t_{s}\right]}\left(1-\frac{y_{s}}{t_{s}}, 1-\frac{z_{s}}{t_{s}^{2}}\right)\right) \rightarrow f(x, y, z), \quad s \rightarrow \infty .
$$

Отсюда получаем существование поточечного предела композиции

$$
\bar{F}_{t_{0, s}, t_{1, s}, s}\left(1-\frac{y_{s}}{t_{s}}, 1-\frac{z_{s}}{t_{s}^{2}}\right) \rightarrow f\left(x_{0}, f\left(x_{1}-x_{0}, y, z\right), 0\right), \quad s \rightarrow \infty .
$$

Функции $\frac{d \bar{F}_{t_{0, s}, t_{1, s}, s}\left(1-\frac{y}{t_{s}}, 1-\frac{z_{s}}{t_{s}^{2}}\right)}{d y}, s=1,2, \ldots$, дифференцируемы при всех $y \geq 0$, а при достаточно больших $s$ их производные ограничены в совокупности:

$$
\left|\frac{\partial^{2} \bar{F}_{t_{0, s}, t_{1, s}, s}\left(1-\frac{y}{t_{s}}, 1-\frac{z_{s}}{t_{s}^{2}}\right)}{\partial y^{2}}\right| \leq \frac{1}{t_{s}} \mathbf{E} \mu_{s}^{(2)}\left(t_{1, s}\right) \leq 2 x_{1} .
$$

Следовательно,

$$
\begin{gathered}
\frac{\partial \bar{F}_{t_{0, s}, t_{1, s}, s}\left(1-\frac{y}{t_{s}}, 1-\frac{z_{s}}{t_{s}^{2}}\right)}{\partial y} \rightarrow \frac{\partial f\left(x_{0}, f\left(x_{1}-x_{0}, y, z\right), 0\right)}{\partial y}, \quad s \rightarrow \infty, \\
\mathbf{E} \mu_{s}\left(t_{1, s}\right)\left(1-\frac{y_{s}}{t_{s}}\right)^{\mu_{s}\left(t_{1, s}\right)-1}\left(1-\frac{z_{s}}{t_{s}^{2}}\right)^{v_{s}\left(t_{0, s}, t_{1, s}\right)} \rightarrow \tilde{\tilde{f}}\left(x_{0}, x_{1}, y, z\right), \quad s \rightarrow \infty,
\end{gathered}
$$

что и требовалось доказать. Теорема доказана. 


\section{5. Характеристики алгоритма при использовании нескольких таблиц}

При использовании $l$ таблиц трудоемкость алгоритма увеличивается в $l$ раз. Размер используемой памяти не меняется. Вычислим надежность алгоритма.

Пусть в алгоритме поиска «золотой» коллизии использовались функции $F_{1}, F_{2}, \ldots, F_{l}$. Через $Z\left(F_{i}\right)$ обозначим подмножество элементов $X$, которые лежат на табличных цепочках метода поиска коллизий функции $F_{i}$, $i \in\{1, \ldots, l\}$.

Множество упорядоченных пар $\left(x_{1}, x_{2}\right)$ элементов множества $Z\left(F_{i}\right)$, удовлетворяющих условиям

$$
F_{i}\left(x_{1}\right)=F_{i}\left(x_{2}\right), \quad \alpha_{i}\left(x_{1}\right)=1, \quad \alpha_{i}\left(x_{2}\right)=2,
$$

обозначим через $K_{i}$.

Множество упорядоченных пар $\left(x_{1}, x_{2}\right) \in X^{2}$, удовлетворяющих условию

$$
\tilde{F}_{1}\left(x_{1}\right)=\tilde{F}_{2}\left(x_{2}\right)
$$

обозначим через $K$.

Пусть упорядоченная пара $k_{0} \in K$ является «золотой» коллизией. Легко заметить, что она будет найдена с помощью описанного выше алгоритма поиска «золотой» коллизии, если выполняется включение

$$
k_{0} \in \bigcup_{i=1}^{l} K_{i}
$$

Рассмотрим среднее значение $\bar{\eta}$ индикатора данного события

$$
\bar{\eta}=\mathbf{E} I\left\{k_{0} \in \bigcup_{i=1}^{l} K_{i}\right\} .
$$

При усреднении будем предполагать, что все множества $K_{i}, i \in\{1, \ldots, l\}$, являются независимыми одинаково распределенными подмножествами $K$, причем любая вероятность вида $\mathbf{P}\left(K_{i}=A\right)$, где $A-$ произвольное подмножество $K$, зависит только от $|A|$. 
Проводя ряд преобразований:

$$
\begin{gathered}
\mathbf{E} I\left\{k_{0} \in \bigcup_{i=1}^{l} K_{i}\right\}=1-\mathbf{E} \prod_{i=1}^{l} I\left\{k_{0} \notin K_{i}\right\}=1-\prod_{i=1}^{l} \mathbf{E} I\left\{k_{0} \notin K_{i}\right\}= \\
=1-\prod_{i=1}^{l}\left(1-\frac{\mathbf{E}\left|K_{i}\right|}{|K|}\right),
\end{gathered}
$$

находим

$$
\bar{\eta}=1-\left(1-\frac{\mathbf{E}\left|K_{1}\right|}{|K|}\right)^{l}
$$

Заметим, что величина $\mathbf{E}\left|K_{1}\right|$ равна среднему количеству найденных коллизий $\left(x_{1}, x_{2}\right)$ функции $F_{1}$, удовлетворяющих дополнительному ограничению $\alpha_{1}\left(x_{1}\right) \neq \alpha_{1}\left(x_{2}\right)$. В силу данного замечания будем считать, что при достаточно больших значениях $q$ величина $\frac{\mathbf{E}\left|K_{1}\right|}{q}$ близка к $\frac{1}{2}$.

Полагая, что свойства отображений $\tilde{F}_{1}(x)$ и $\tilde{F}_{2}(x)$ подобны свойствам случайных отображений множества $X$ в себя, будем считать, что при достаточно больших значениях $N$ величина $\frac{|K|}{N}$ близка к 1 .

В качестве надежности $\eta$ алгоритма поиска «золотой» коллизии возьмем величину, полученную из (18) путем замены выражения $\frac{\mathbf{E}\left|K_{1}\right|}{|K|}=\frac{\mathbf{E}\left|K_{1}\right|}{q}$. $\frac{N}{|K|} \cdot \frac{q}{N}$ на $\frac{q}{2 N}$. В результате получим

$$
\eta=1-\left(1-\frac{q}{2 N}\right)^{l}=1-\left(1-\gamma_{1}\left(\alpha, x_{0}, x_{1}\right) \frac{m}{2 N}\right)^{l}
$$

Итоговые значения характеристик алгоритма поиска «золотой» коллизии отразим в следующей таблице.

\begin{tabular}{|l|l|}
\hline Характеристика & $\begin{array}{l}\text { Выражение характеристики } \\
\text { через параметры }\end{array}$ \\
\hline Надежность $\eta$ & $1-\left(1-\gamma_{1}\left(\alpha, x_{0}, x_{1}\right) \frac{m}{2 N}\right)^{l}, \alpha=\frac{m t^{2}}{N} \cdot \frac{1}{2}$ \\
\hline Объем памяти $M$ & $m \gamma_{0}\left(x_{0}, x_{1}\right)$ \\
\hline $\begin{array}{l}\text { Суммарная трудоемкость } T \\
\left(T_{0}+T_{1}\right)\end{array}$ & $m t l\left(\gamma_{2}\left(x_{1}\right)+\gamma_{3}\left(\alpha, x_{0}, x_{1}\right)\right)$ \\
\hline
\end{tabular}

Формулы для множителей $\gamma_{0}\left(x_{0}, x_{1}\right), \gamma_{1}\left(\alpha, x_{0}, x_{1}\right), \gamma_{2}\left(x_{1}\right), \gamma_{3}\left(\alpha, x_{0}, x_{1}\right)$ приведены в формулировке теоремы 1. 


\section{Список литературы}

[1] Oorschot P.C., Wiener M.J. "Parallel collision search with cryptanalytic applications", J. Cryptology, 12 (1999), 1-28.

[2] Oorschot P.C., Wiener M.J. "Parallel collision search with application to hash functions and discrete logarithms" // 2nd ACM Conf. on Computer and Commun. Security, Fairfax, Virginia, 1994, 210-218.

[3] Oorschot P.C., Wiener M.J. "Improving implementable meet-in-the-middle attacks by orders of magnitude", CRYPTO'96, Lect. Notes Comput. Sci., 1109, 1996, 229-236.

[4] Borst J., Preneel B., Vandewalle J., "On the time-memory tradeoff between exhaustive key search and table precomputation", Proc. 19th Symp. Inf. Theory in the Benelux, Veldhoven, Netherlands, 1998, 111-118.

[5] Standaert F.X., Rouvroy G., Quisquater J.J., Legat J.D. “A time-memory tradeoff using distinguished points: New analysis \& FPGA results", Proc. CHES 2002, Lect. Notes Comput. Sci., 2523, 2003, 596-611.

[6] Pilshchikov D.V. "Estimation of the characteristics of time-memory-data tradeoff methods via generating functions of the number of particles and the total number of particles in the Galton-Watson process", Математические вопросы криптографuи, 5:2 (2014), 103-108.

[7] Pilshchikov D.V. "On the limiting mean values in probabilistic models of timememory-data tradeoff methods", Математические вопросы криптографии, 6:2 (2015), 59-65. 